
КОНСТИТУЦИОНАЛЬНОМ ОЖИРЕНИИ

(с М.Б. Лясникова, Н.А. Белякова*, И.Г. Цветкова, А.А. Родионов, Н.О. Милая

Тверской государственный медицинский университет, Тверь, Россия

Обоснование. Алиментарно-конституциональное ожирение, ввиду его высокой распространенности, является ключевой проблемой современной медицины. Однако не всякое ожирение сопровождается развитием метаболических нарушений, приводящих к ранней инвалидизации и смертности. В связи с этим актуальным является изучение рисков метаболического нездоровья при ожирении.

Цель. Выявить факторы, повышающие риск развития метаболического нездоровья при алиментарно-конституциональном ожирении.

Методы. У пациентов с алиментарно-конституциональным ожирением проведены оценка антропометрических данных (индекса массы тела, объемов талии и бедер и их соотношения), уровня артериального давления, результатов лабораторных исследований (глюкоза, инсулин, индексы инсулинорезистентности, лептин, холестерин и фракции, триглицериды, аспартатаминотрансфераза, аланинаминотрансфераза, гамма-глутамилтрансфераза), состава тела методом биоимпеданса, анкетирование по особенностям питания и физической активности.

Результаты. В зависимости от метаболического здоровья пациенты были разделены на две группы: основная

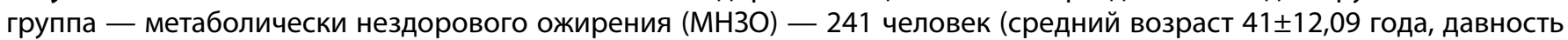

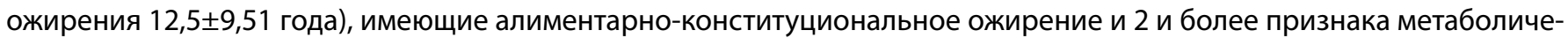

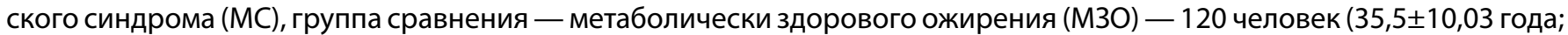
$\mathrm{p}<0,05,8,0 \pm 7,39$ года; $\mathrm{p}<0,05$ соответственно) с алиментарно-конституциональным ожирением и одним признаком МС или без них. Анализ изученных факторов риска формирования метаболического нездоровья при алиментарно-конституциональном ожирении показал, что наибольшее значение для развития МНЗО имеет абдоминальное ожирение (увеличение окружности талии у женщин более 88 см и у мужчин более 102 см). При этом МНЗО ассоциировано не только с классическими признаками МС, но и с уровнем инсулина крови, инсулинорезистентностью, нарушениями липидного спектра и функции печени. Наибольший риск появления метаболических нарушений имеют пациенты в возрасте старше 45 лет, со снижением активной клеточной массы в организме менее 45\%, давностью ожирения более 10 лет и отягощенной по данной патологии наследственностью. Среди поведенческих факторов (пищевой статус) риск метаболического нездоровья при ожирении повышают жирные молочные продукты, а частые перекусы, сладкие безалкогольные напитки, напротив, не влияют на него.

Заключение. Формирование МНЗО ассоциировано не только с возрастом пациента, длительностью ожирения, показателями углеводного и липидного обменов, но также со снижением процента метаболически активных тканей организма и поведенческими факторами.

КЛЮЧЕВЫЕ СЛОВА: ожирение; метаболический синдром; метаболические нарушения; поведенческие факторы риска.

\title{
RISKS FOR DEVELOPMENT OF METABOLIC DISORDERS IN ALIMENTARY CONSTITUTIONAL OBESITY
}

○ Mariya B. Lyasnikova, Nataliya A. Belyakova*, Inna G. Tsvetkova, Andrey A. Rodionov, Nataliya O. Milaya

Tver State Medical University, Tver, Russia

BACKGROUND: alimentary-constitutional obesity due to it's high prevalence, is the key problem of modern healthcare system. However, obesity is not always accompanied with metabolic disorders, leading to early invalidization and mortality. That's why it is important to study risks of metabolical nonhealth in obesity.

AIM: to detect factors, increasing risks of development of metabolic disbalance in alimentary-constitutional obesity.

MATERIALS AND METHODS: In patients with alimentary-constitutional obesity there was performed an examination including anthropometry (body mass index, Waist Circumference, Hip Circumference,waist to hip ratio), blood pressure measurement, laboratory tests - metabolic indexes: glucose, insulin, insulin resistance indexes, leptin, cholesterol, cholesterol of lipoproteins, triglycerides, aspartate aminotransferase, alanine aminotransferase, gamma-glutamiltransferase), body composition measurement by bioelectrical impedance analysis; patients were also interviewed on their behavior (food habits) and physical activity.

RESULTS: There were formed two groups depending on metabolic health indexes: main group - metabolically non-healthy obesity (MNHO) - 241 persons (aged 41 $\pm 12,09$, duration of obesity 12,5 $\pm 9,51$ years) with alimentary-constitutional obesity and two or more signs of MS, a comparison group - of metabolically healthy obesity (MHO) - 120 persons (aged 35,5 $\pm 10,03$; 
$p<0,05$, duration of obesity $8,0 \pm 7,39$ years; $p<0,05$ ) with alimentary-constitutional obesity and one sign of MS or without it. Data analysis of studied risk factors for development of metabolically non-healthy alimentary-constitutional obesity confirmed that most relevant factor in development of MNHO is abdominal fat mass distribution (increasing of Waist Circumference over $88 \mathrm{sm}$ in females and over $102 \mathrm{sm}$ in mails). At the same time MNHO had correlation not only with classical signs of MS, but also with blood insulin level, insulin resistance indexes, fat metabolism disbalance and liver disfunction. More severed risk for appearance of metabolic disorders have patients over 45 years old with decreased active cell mass (less than $45 \%$ ), duration of obesity above 10 years and obesity-burdened heredity. In food habits risk of development of metabolically nonhealthy obesity was increased in taking of fat milk food, and, on the contrary, - frequent snacks, alcohol free sweet drinks didn't affect it.

CONCLUSION: Development of MNHO is associated not only with the age of patient, duration of obesity, carbohydrate and fat metabolism indexes, but also with decreased percentage of metabolically active tissues and some food habits.

KEYWORDS: obesity; metabolic syndrome; metabolic disorders; behavior risk factors.

\section{ОБОСНОВАНИЕ}

Прогрессирующий рост распространенности алиментарно-конституционального ожирения и несомненная его взаимосвязь с сахарным диабетом 2 типа, сердечно-сосудистыми заболеваниями и некоторыми видами рака заставили научное сообщество признать ожирение одной из ключевых проблем современной медицины [1, 2]. В основе диагностики избыточной массы тела и ожирения лежит индекс массы тела (ИМТ), простой и удобный в практическом применении показатель, величина которого коррелирует с развитием кардиоваскулярных событий. По данным The Global BMI Mortality Collaboration, ИМТ 20-25 кг/м² ассоциирован с самым низким уровнем смертности в популяции [3]. Однако, как показывают современные научные исследования, нозология «ожирение», основанная лишь на ИМТ, представлена неоднородной группой пациентов с разным метаболическим профилем [4, 5]. Современные данные говорят о том, что метаболические нарушения и кардиоваскулярные риски при ожирении в большей степени связаны с локализацией жировой ткани и нарушением функционирования адипоцитов, нежели с общим содержанием жира в организме, и именно эти параметры являются более точными предикторами формирования вышеуказанных рисков [6-8]. В ходе исследования NHANES были получены данные, свидетельствующие о том, что 51,3\% людей с избыточной массой тела и 31,7\% с ожирением являются метаболически здоровыми [9]. В свете концепции метаболического нездоровья особый интерес представляют факторы риска, связанные с его возникновением.

\section{ЦЕЛЬ ИССЛЕДОВАНИЯ}

Выявить факторы, повышающие риск развития метаболического нездоровья при алиментарно-конституциональном ожирении.

\section{МАТЕРИАЛЫ И МЕТОДЫ}

\section{Место и время проведения исследования}

Место проведения. Работа проведена на базе амбулаторного приема частной клиники «Восстановительная медицина» (главный врач - Е.Б. Максубова) и клиники Тверского государственного медицинского университета (главный врач - к.м.н. Е.Е. Пичуев).

Время исследования. С декабря 2010 г. по январь 2017 г.
Изучаемые популяции (одна или несколько)

Изучалась одна популяция.

Критерии включения: диагностированное эндокринологом алиментарно-конституциональное ожирение или избыточная масса тела, возраст от 18 до 60 лет.

Критерии исключения: острые инфекционные заболевания, ранее диагностированные хронические заболевания в стадии обострения, декомпенсации, сердечно-сосудистая патология с тяжелой сердечной недостаточностью, сахарный диабет при потребности в инсулинотерапии, хронические вирусные гепатиты $(B, C)$, алкогольное поражение печени.

Способ формирования выборки из изучаемой

популяции (или нескольких выборок из нескольких

изучаемых популяций)

Выборка формировалась из пациентов, обратившихся к эндокринологу по поводу избыточного веса. Группы были сформированы сплошным включением наблюдений.

\section{Дизайн исследования}

Одноцентровое интервенционное поперечное одномоментное одновыборочное сравнительное исследование.

Описание медицинского вмешательства

(для интервенционных исследований)

Дополнительно к стандартному обследованию пациента с ожирением у эндокринолога выполнялись: оценка состава тела (СТ), психологическое тестирование, включая изучение пищевого поведения и анкетирование по особенностям питания и физическим нагрузкам.

Методы

В исследование включались все пациенты, обратившиеся на прием к эндокринологу по поводу избыточной массы тела, имеющие ИМТ более 25, в возрасте от 18 до 60 лет.

Критерии включения в подгруппы (метаболически здоровое (М3О) и метаболически нездоровое (МН3О) ожирение) оценивались эндокринологом на основе жалоб, анамнестических данных, объективного обследования и результатов лабораторных исследований, относящихся к критериям метаболического синдрома: определяли объем талии, уровни триглицеридов, холестерина липопротеидов высокой плотности (ХС ЛПВП), глюкозы натощак, проводили измерение артериального давления (АД).

Критерии исключения выявлялись на основании сбора анамнеза, объективных данных и анализа имеющейся 
медицинской документации (ранее выполненных консультаций терапевта, кардиолога, инфекциониста, психиатра).

Клинический осмотр проводился с применением общепринятых методов физикального обследования с учетом предъявляемых жалоб. Измерение АД (мм рт. ст.) осуществлялось при помощи тонометра со встроенным в манжету фонендоскопом. Измерение проводилось трижды в спокойной обстановке, после пятиминутного отдыха. Регистрировали среднее значение. Рост (см) измерялся с помощью станкового ростомера с точностью до 0,5 см. Масса тела (кг) измерялась на медицинских весах с погрешностью $\pm 0,05$ кг. Оценка избыточности питания проводилась по отношению массы тела к росту в квадрате (ИМТ) по критериям ВОЗ (1997) [10]. Для оценки типа распределения жировой ткани (абдоминальный или гиноидный тип) проводилось измерение гибкой сантиметровой лентой окружности талии (ОТ, см) и окружности бедер (ОБ) по общепринятой методике, с расчетом отношения ОТ/ОБ.

Для лабораторного анализа у пациентов утром натощак забиралась венозная кровь из кубитальной вены в количестве 5 мл в вакуумную пробирку. Само исследование проводилось на автоматизированной модульной платформе Roche Cobas 8000 с биохимическим модулем с702, Roche Diagnostics, Швейцария. Для этого использовались следующие методы: ферментативно-колориметрический (липиды и гамма-глутамилтранспептидаза (Гамма-ГТ)), кинетический метод без пиридоксаль-5-фосфата (аспартатаминотрансфераза (АСТ) и аланинаминотрансфераза (АЛТ)), ферментативно-гексокиназный (глюкоза), иммунохемилюминесцентный (инсулин) и иммуноферментный (лептин) без анализатора. Инсулинорезистентность (ИР) изучалась с помощью гомеостатической модели HOMА [(глюкозахинсулин)/22,5] и индекса Caro (глюкоза/ инсулин). Интерпретация лабораторных данных осуществлялась по референсным значениям: для холестерина плазмы (ХС, референтный интервал 3,2-5,2 ммоль/л), липопротеидов низкой плотности (ХС ЛПНП, 0,0-3,5 ммоль/л), липопротеидов очень низкой плотности (ХС ЛПОНП, 0,0-0,9 ммоль/л), ХС ЛПВП, >1,3 ммоль/л, триглицеридов (ТГ, <1,7 ммоль/л), лептина плазмы (1,1-27,6 нг/мл), глюкозы плазмы (ГЛ, <6,1 ммоль/л) и инсулина (2,6-24,9 мкЕД/мл), с расчетом индексов ИР: HOMA-IR (норма <2,55 ед), Caro-IR (норма >0,33 ед); а также АСТ (до 45 MЕ/л), АЛТ (до 40 MЕ/л) и Гамма-ГТ (до $35 \mathrm{ME} / л)$.

Диагностика МС основывалась на нормативных критериях NCEP ATP III (2001) [11], к которым относится абдоминальное ожирение (ОТ: у женщин $>88 \mathrm{~cm}$, у мужчин $>102$ см);ТГ> 1,7 ммоль/л,ХСЛПВП:уженщин <1,3 ммоль/л, у мужчин <1,04 ммоль/л; АД $\geq 130 / 85$ мм рт. ст., уровень глюкозы натощак $\geq 6,1$ ммоль/л [12]. М3О считали такое, при котором женщины и мужчины не имели ни одного критерия МС или имели только один признак по NCEP ATP III (2001). Для МНЗО было характерно наличие двух и более критериев МС [13].

СТ изучался с помощью биоэлектрического импедансного метода, который заключался в измерении сопротивления тканей организма до и после воздействия переменным током различной частоты. Для этого использовался анализатор СТ - ЗАО «Диамант», прибор 8804. Исследование проводилось в утренние часы. Электроды устанавливались плотно, дистально, на обе голени и оба предплечья. С наложенными электродами обследуемый находился в положении лежа не менее 10 мин и затем 2-3 мин длилось само исследование. В конце автоматически формировалось заключение, которое включало абсолютные и относительные значения следующих показателей: жировой массы (ЖМ, кг, \%), общей жидкости (ОЖ, л), общей воды (OВ, л, \%) - внеклеточной (ВКЖ, л), внутриклеточной жидкости (ВнКЖ, л), безжировой массы (БЖМ, кг, \%), активной клеточной массы (АКМ, кг, \%), сухой клеточной массы (СКМ, кг, \%).

Психологическое тестирование проводилось для диагностики тревожно-депрессивных нарушений (совместно с психотерапевтом): по шкале Бека (депрессия легкая - 10-15 баллов, умеренная - 16-19, выраженная - 20-29, тяжелая - 30 и более баллов) [14] и шкале Спилбергера, адаптированной Ю.Л. Ханиным (PX1 - ситуативная или реактивная и PX2 - личностная тревога) с градациями «низкая», «умеренная» и «высокая» [15]. Также изучались особенности пищевого поведения с помощью опросника, созданного T. Van Strien, включавшего 33 утверждения, каждое из которых респондент должен был оценить в отношении себя как «Никогда», «Редко», «Иногда», «Часто» и «Очень часто». В нашей анкете вопросы 1-10 относились к экстернальному пищевому поведению (более 2,7 балла), вопросы 11-23 - эмоциональному (более 1,8 балла), вопросы 24-33 - ограничительному пищевому поведению (более 2,4 балла) [16].

Кроме этого, всем обследованным провели анкетирование по особенностям питания и физическим нагрузкам [17]. Анкета включала 31 вопрос о возможных причинах наличия избытка массы тела у обследуемых, мотивах, побуждающих к его снижению, особенностях питания и семейных привычках, физической активности. Пациент выбирал один или несколько ответов по своему усмотрению. В анализ вошли вопросы, позволяющие оценить особенности питания, данные пищевого анамнеза (в детском возрасте), а также физическую активность респондента.

\section{Анализ в подгруппах}

Группы формировались в зависимости от метаболического здоровья, на основании критериев NCEP ATP III (2001) [11]. Согласно им, в понятие метаболического синдрома (МС) входят: абдоминальное ожирение (ОТ у мужчин >102 см, женщин >88 см), триглицериды $>$ 1,7 ммоль/л, ХС ЛПВП (у мужчин <1,04 ммоль/л, у женщин <1,3 ммоль/л), АД >130/85 мм рт. ст., уровень глюкозы натощак $>6,1$ ммоль/л. М3О мы считали ожирение у женщин, которые не имели ни одного критерия МС или только один по NCEP ATP III (2001). Тех же, у кого было зафиксировано более одного признака, относили к МНЗО.

\section{Этическая экспертиза}

Данное исследование прошло согласование и одобрение в комиссии Этического комитета Тверского государственного медицинского университета (протокол № 9 от 30 ноября 2015 г.). Все принимающие участие были в полной мере проинформированы о характере и целях исследовательской работы, предполагаемых процедурах, вероятном риске, ожидаемом положительном эффекте, о полностью добровольном участии и возможности прекращения участия в исследовании в любое время без объяснения причин. Все обследованные подписывали информированное добровольное согласие. 


\section{Статистический анализ}

Статистическая обработка данных проводилась с использованием пакета прикладных статистических программ Statistica 10. Результаты представлены в виде среднего значения и стандартного отклонения $(\mathrm{M} \pm \sigma)$. Данные исследовались на нормальность, после чего принималось решение об использовании конкретного статистического метода. Статистическая значимость межгрупповых различий оценивалась с помощью критериев: Стьюдента (t) - в случае нормального распределения, в противном случае - Манна-Уитни (U) для двух групп. Для качественного сравнительного анализа применялся хи-квадрат $\left(\mathrm{X}^{2}\right)$, а корреляционные взаимосвязи изучались с помощью критерия Пирсона $\left(\mathrm{R}_{\mathrm{xy}}\right)$ и ранговой корреляции Спирмена $\left(\mathrm{R}_{\mathrm{s}}\right)$. Для определения сравнительных рисков развития ассоциированных с ожирением состояний использовался метод расчета отношения шансов (ОШ) с 95\% доверительным интервалом (ДИ) (ОШ $>1,0$ указывало на наличие риска появления того или иного признака при условии, что 95\% ДИ не содержит 1). Для количественной оценки вероятности исхода, связанного с наличием фактора риска (АКМ\%), использовался расчет относительного риска (ОР) с 95\% ДИ. Критический уровень значимости при проверке статистических гипотез - $\mathrm{p}<0,05$.

\section{РЕЗУЛЬТАТЫ}

\section{Объекты (участники) исследования}

Из числа включенных в исследование обратившихся в клинику для коррекции веса (361) большинство женщины - 92\%. Все пациенты были разделены на две группы: основная группа - МН3О - 241 человек (мужчин 28,

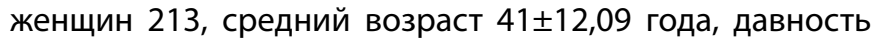

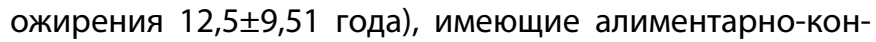
ституциональное ожирение и два и более признака МС. В группу сравнения - М3О - вошли 120 человек (мужчин 2, женщин $118,35,5 \pm 10,03$ года; $8,0 \pm 7,39$ года; все $\mathrm{p}<0,05)$ с алиментарно-конституциональным ожирением и одним признаком МС или без них.

\section{Основные результаты исследования}

Была проанализирована распространенность МС в нашей когорте пациентов. Из признаков МС наиболее часто диагностировалось увеличение ОТ (80,6\%), в половине случаев - АГ $(53,9 \%)$ и снижение уровня ХС лПВП (50\%) и реже всего - повышение уровней глюкозы $(31,2 \%)$ и ТГ $(26,7 \%)$.

В таблице 1 дана клинико-лабораторная характеристика выделенных групп обследованных.

Следует отметить, что у пациентов с МН3О были выше, чем у обследованных с МЗО, ИМТ, ОТ/ОБ, а также не только критерии МС (ОТ, АД, ТГ, ХС ЛПВП, глюкоза; все $\mathrm{p}<0,05)$, но и другие метаболические показатели, отражающие липидный профиль, уровни инсулина и лептина, ИР, функцию печени (все $\mathrm{p}<0,05)$, хотя большинство из них находилось в референсном диапазоне. Учитывая это, представляло интерес оценить риск для метаболического нездоровья изучаемых показателей.

Корреляционный анализ проводился раздельно в группах МН3О и М3О. Ниже приводятся данные, показавшие взаимосвязь между показателями в обеих группах. Так, возраст и ИМТ имели слабую корреляцию с уровнем холестерина: при $\mathrm{MH} 3 \mathrm{O} \mathrm{R}_{\mathrm{xy}}=0,27,0,26 ; \mathrm{p}<0,001$ и при M3О $\mathrm{R}_{x y}=0,19,0,17 ; \mathrm{p}<0,05$. ИМТ, в свою очередь, имел взаимосвязь со многими показателями: высокую

Таблица 1. Результаты клинико-лабораторного обследования пациентов с ожирением в зависимости от метаболического здоровья $(\mathrm{M} \pm \sigma)$

\begin{tabular}{|c|c|c|c|}
\hline Показатель & Пациенты с МH30, n=241 & Пациенты с М30, n=120 & $\mathbf{P}$ \\
\hline 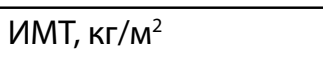 & $36,2 \pm 6,66$ & $30,8 \pm 4,56$ & $\mathrm{p}<0,0001$ \\
\hline OT, см & $106,1 \pm 15,52$ & $92,2 \pm 10,49$ & $\mathrm{p}<0,0001$ \\
\hline ОТ/ОБ & $0,87 \pm 0,10$ & $0,81 \pm 0,08$ & $\mathrm{p}<0,0001$ \\
\hline САД, мм рт. ст. & $132,2 \pm 16,75$ & $114,4 \pm 11,37$ & $\mathrm{p}<0,0001$ \\
\hline ДАД, мм рт. ст. & $85,5 \pm 10,61$ & $75,6 \pm 7,83$ & $\mathrm{p}<0,009$ \\
\hline Глюкоза, ммоль/л & $5,5 \pm 1,12$ & $4,9 \pm 0,48$ & $\mathrm{p}<0,0001$ \\
\hline Инсулин, мкЕД/мл & $17,1 \pm 11,07$ & $9,82 \pm 4,31$ & $\mathrm{p}<0,0001$ \\
\hline HOMA-IR, ед & $4,26 \pm 3,230$ & $2,12 \pm 0,970$ & $\mathrm{p}<0,0004$ \\
\hline Caro-IR, ед & $0,44 \pm 0,27$ & $0,59 \pm 0,25$ & $\mathrm{p}<0,0003$ \\
\hline ТГ, ммоль/л & $1,67 \pm 0,94$ & $1,00 \pm 0,41$ & $\mathrm{p}<0,0001$ \\
\hline ХС, ммоль/л & $5,6 \pm 1,17$ & $5,2 \pm 1,14$ & $\mathrm{p}<0,001$ \\
\hline ХС ЛПНП, ммоль/л & $3,68 \pm 1,11$ & $3,2 \pm 1,24$ & $\mathrm{p}<0,005$ \\
\hline ХС ЛПОНП, ммоль/л & $0,83 \pm 0,55$ & $0,57 \pm 0,25$ & $p<0,005$ \\
\hline ХС лПВП, моль/л & $1,24 \pm 0,31$ & $1,49 \pm 0,38$ & $\mathrm{p}<0,0001$ \\
\hline Лептин, нг/мл & $38,7 \pm 23,81$ & $28,7 \pm 17,78$ & $p<0,001$ \\
\hline АСТ, МЕ/л & $26,1 \pm 18,58$ & $20,5 \pm 7,69$ & $\mathrm{p}<0,02$ \\
\hline АЛТ, МЕ/л & $31,7 \pm 35,24$ & $21,2 \pm 13,41$ & $\mathrm{p}<0,02$ \\
\hline Гамма-ГТ, МЕ/л & $46,4 \pm 64,09$ & $23,8 \pm 20,21$ & $\mathrm{p}<0,05$ \\
\hline
\end{tabular}




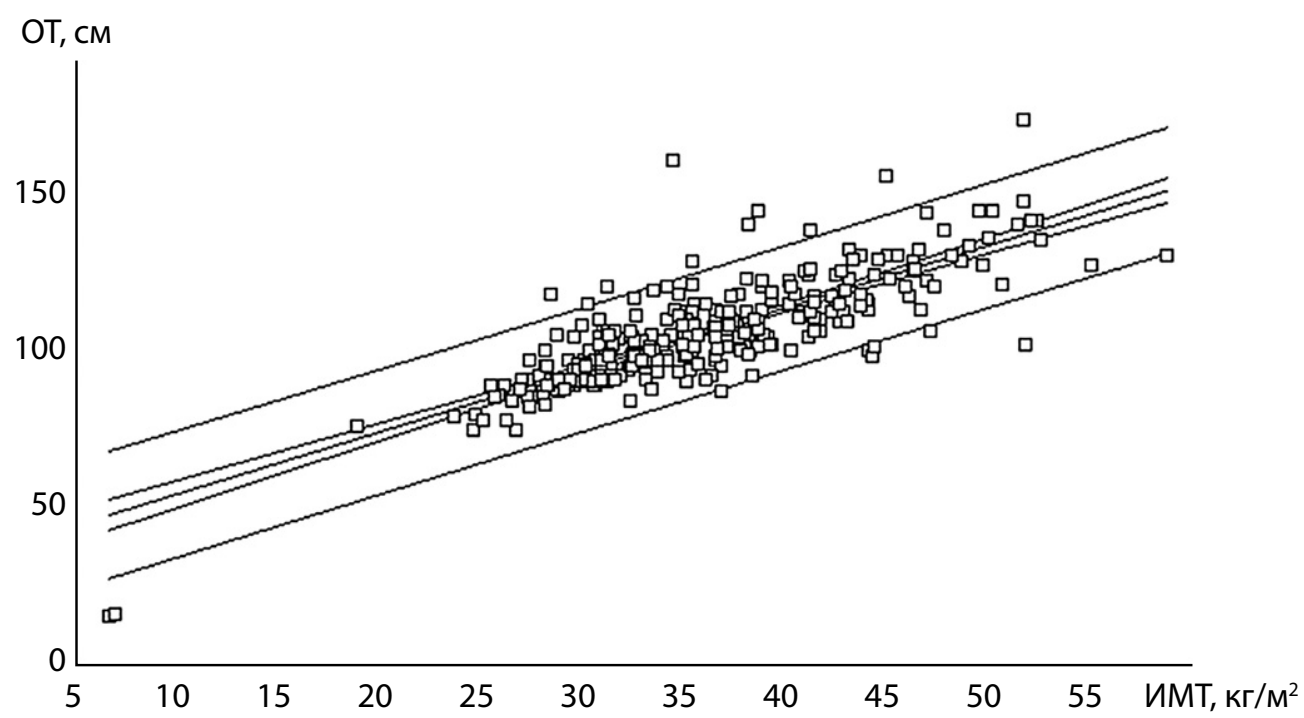

Рисунок 1. Взаимосвязь между индексом массы тела (ИМТ) и окружностью талии (ОТ) у пациентов с метаболически нездоровым ожирением.

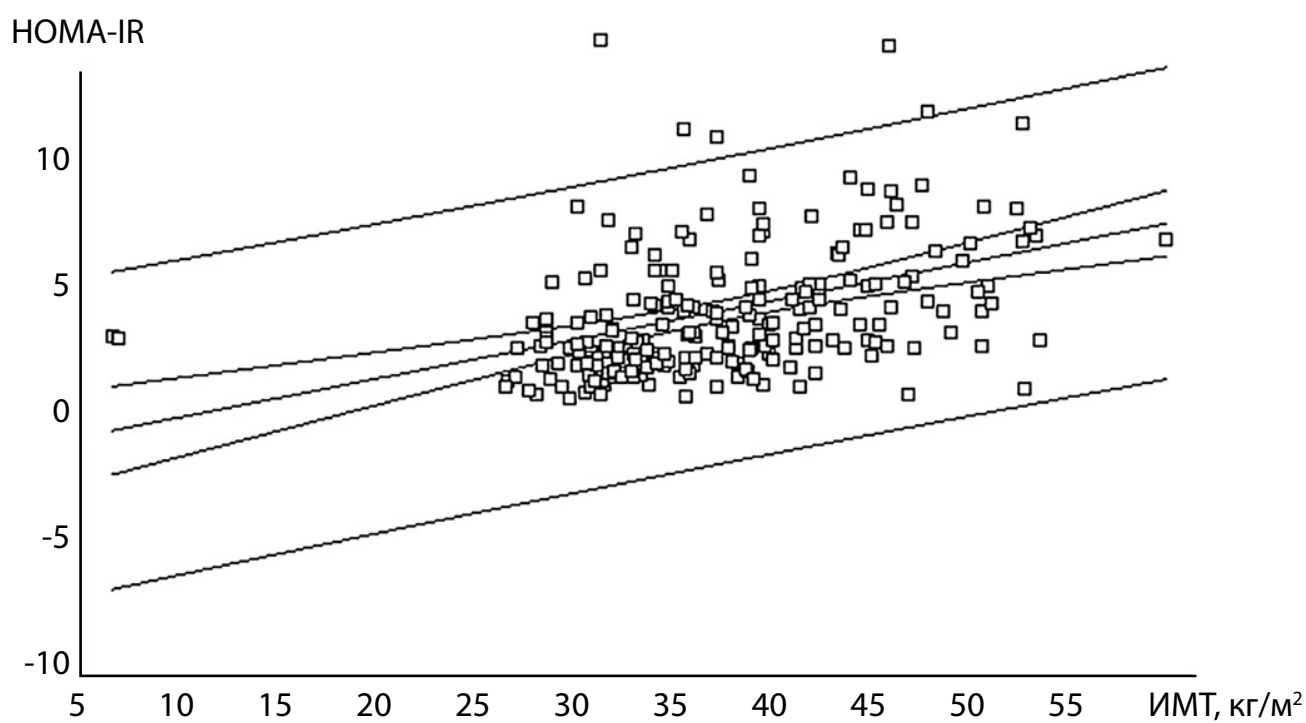

Рисунок 2. Взаимосвязь между индексом массы тела и показателем инсулинорезистентности (HOMA-IR) у пациентов с метаболически нездоровым ожирением.

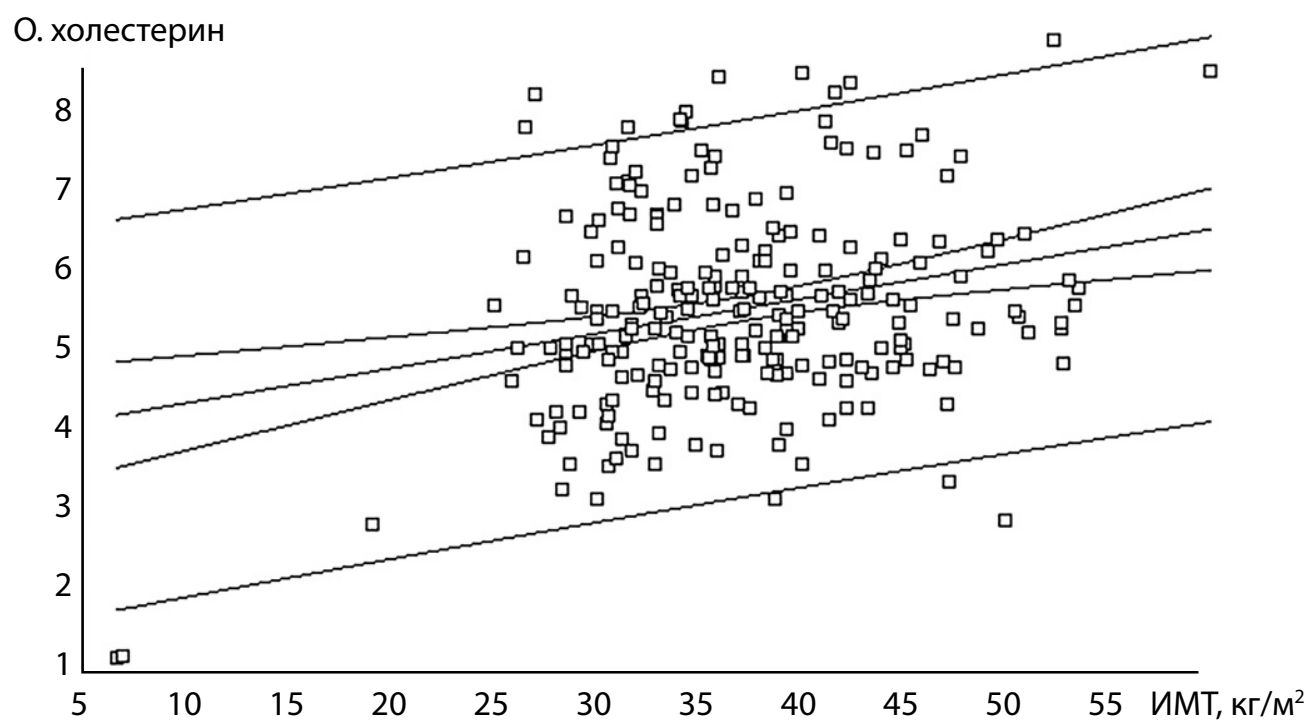

Рисунок 3. Взаимосвязь между индексом массы тела и холестерином плазмы крови у пациентов с метаболически нездоровым ожирением. 
с ОТ (соответственно $\mathrm{R}_{\mathrm{xy}}=0,81,0,80 ; \mathrm{p}<0,001$ ), слабую с ДАД $\left(R_{x y}=0,21,0,19 ; p<0,05\right)$, умеренную с инсулином и ИР при МН3О ( $\left.\mathrm{R}_{\mathrm{xy}}=0,37,0,34 ; \mathrm{p}<0,001\right)$ и слабую при $\mathrm{M} 3 \mathrm{O}$ $\left(R_{x y}=0,24,0,25 ; p<0,02\right)$ (рис. 1-3).

ИР имела очень высокую корреляцию с уровнем инсулина (соответственно $\mathrm{R}_{x y}=0,95,0,96 ; \mathrm{p}<0,001$ ) и умеренную с глюкозой $\left(R_{x y}=0,46,0,3 ; p<0,002\right)$, слабую с лептином при $\mathrm{MH} 30\left(\mathrm{R}_{x y}=0,17 ; \mathrm{p}<0,05\right)$ и умеренную при $\mathrm{M} 30$ $\left(\mathrm{R}_{\mathrm{xy}}=0,32 ; \mathrm{p}<0,02\right)$, а также слабую с Гамма-ГТ $\left(\mathrm{R}_{\mathrm{xy}}=0,17\right.$, $0,21 ; p<0,05)$. Проведенный корреляционный анализ позволяет сделать вывод, что большинство метаболических нарушений обусловлено степенью избыточной массы тела.

Анализ состава тела подтвердил соответствие его результатов данным клинического исследования. При МНЗО были выше, чем при М3О, все абсолютные показатели: ЖМ (соответственно 37,6 $\pm 11,08$ и 30,2 $\pm 8,73$ кг; $\mathrm{p}<0,05), O B(44,0 \pm 7,67$ и 38,4 $44,74 \pi ; \mathrm{p}<0,05)$, ОЖ $(37,2 \pm 5,46$

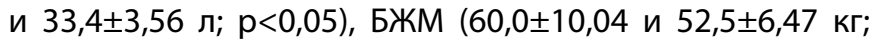
$\mathrm{p}<0,05), \operatorname{CKM}(10,1 \pm 1,75$ и $8,8 \pm 1,11 к г ; p<0,05)$ и АКМ $(40,1 \pm 6,88$ и 34,4 4,34 кг; $p<0,05)$. Достоверных различий между группами в процентах параметров состава тела не было. Несмотря на это, далее был проведен корреляционный анализ. Так, он показал, что ранговая корреляция между МHЗО и МЗО по ЖМ \% не имела достовер- ных различий (Rs=-0,02; p>0,05); а вот взаимосвязь БЖМ \% с развитием МНЗО была слабой $(R s=0,27 ; \mathrm{p}<0,001)$, a с AKM \% - умеренной (Rs=0,32; $\mathrm{p}<0,001)$.

Нарушения пищевого поведения диагностировались в 59\% случаев, при этом достоверных различий между группами не было. Средние баллы по экстернальному и эмоциональному пищевому поведению также не отличались, а вот по ограничительному типу балл был выше

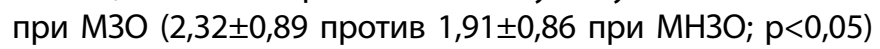
и соответствовал норме, в то время как при $\mathrm{MH} 30$ он свидетельствовал о приеме пище большинством пациентов без ограничений. Достоверных различий в психологических тестах не отмечено. В обеих группах преобладала легкая депрессия (32,1\% при М3О и 33,9\% при МН3О), выраженная личностная тревога (соответственно в 77,6 и 76,4\% случаев) и умеренная ситуативная (в 78,9 и $74,8 \%$ случаев).

В таблице 2 приводятся только достоверные результаты анкетирования по пищевому статусу и физическим нагрузкам. Согласно представленным данным, женщины с МН3О чаще использовали в своем рационе животные белки (мясо, птица, рыба), жирные молочные продукты и реже - овощи и фрукты. В то же время женщины с М3О чаще перекусывали и употребляли кофе, а вот физические нагрузки у них были реже.

Таблица 2. Данные анкетирования по питанию и физическим нагрузкам пациентов с ожирением в зависимости от метаболического здоровья, $\mathrm{n}, \%$

\begin{tabular}{|c|c|c|c|c|c|}
\hline \multirow{2}{*}{ Переменная } & \multicolumn{2}{|c|}{ MH30, n=241 } & \multicolumn{2}{|c|}{$M 30, n=120$} & \multirow{2}{*}{$\mathbf{P}$} \\
\hline & $\mathbf{n}$ & $\%$ & $\mathbf{n}$ & $\%$ & \\
\hline \multicolumn{6}{|l|}{ Перекус } \\
\hline обычно & 94 & $39 \%$ & 54 & $45 \%$ & \multirow{3}{*}{$<0,001$} \\
\hline редко & 58 & $24 \%$ & 44 & $37 \%$ & \\
\hline никогда & 89 & $37 \%$ & 22 & $18 \%$ & \\
\hline \multicolumn{6}{|l|}{ Мясо, рыба, птица } \\
\hline ежедневно & 182 & $76 \%$ & 71 & $59 \%$ & \multirow{3}{*}{0,002} \\
\hline еженедельно & 46 & $19 \%$ & 44 & $37 \%$ & \\
\hline редко & 12 & $5 \%$ & 5 & $4 \%$ & \\
\hline \multicolumn{6}{|l|}{ Овощи и фрукты } \\
\hline ежедневно & 123 & $51 \%$ & 80 & $67 \%$ & \multirow[t]{2}{*}{0,003} \\
\hline еженедельно и реже & 118 & $49 \%$ & 40 & $33 \%$ & \\
\hline \multicolumn{6}{|l|}{ Потребляемые молочные продукты } \\
\hline жирные & 106 & $44 \%$ & 28 & $23 \%$ & \multirow[t]{2}{*}{$<0,001$} \\
\hline обезжиренные & 135 & $56 \%$ & 92 & $77 \%$ & \\
\hline \multicolumn{6}{|c|}{ Предпочитаемые безалкогольные напитки: } \\
\hline кофе & 118 & $49 \%$ & 68 & $57 \%$ & 0,102 \\
\hline чай/чай, кофе & 174 & $72 \%$ & 94 & $78 \%$ & 0,001 \\
\hline газированная вода, соки & 53 & $22 \%$ & 42 & $35 \%$ & 0,006 \\
\hline \multicolumn{6}{|l|}{ Физическая активность } \\
\hline ежедневно & 159 & $66 \%$ & 53 & $44 \%$ & \multirow{3}{*}{0,001} \\
\hline от случая к случаю & 39 & $16 \%$ & 40 & $33 \%$ & \\
\hline не занимаюсь & 43 & $18 \%$ & 27 & $23 \%$ & \\
\hline
\end{tabular}


старше 45 лет

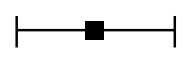

мужской пол

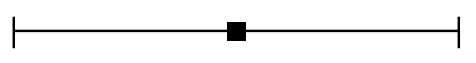

наследственность

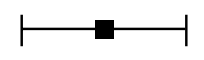

давность

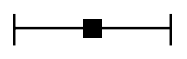

ИМТ выше 35

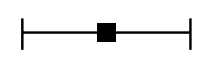

OT/OБ

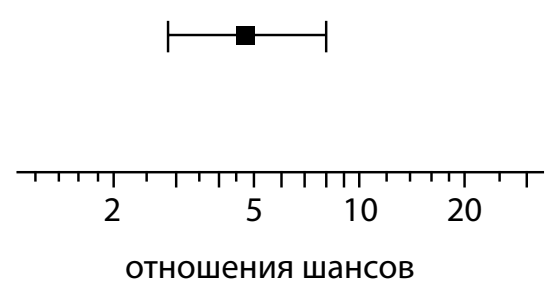

Рисунок 4. Анамнестические и объективные данные, повышающие риск диагностики метаболически нездорового ожирения.

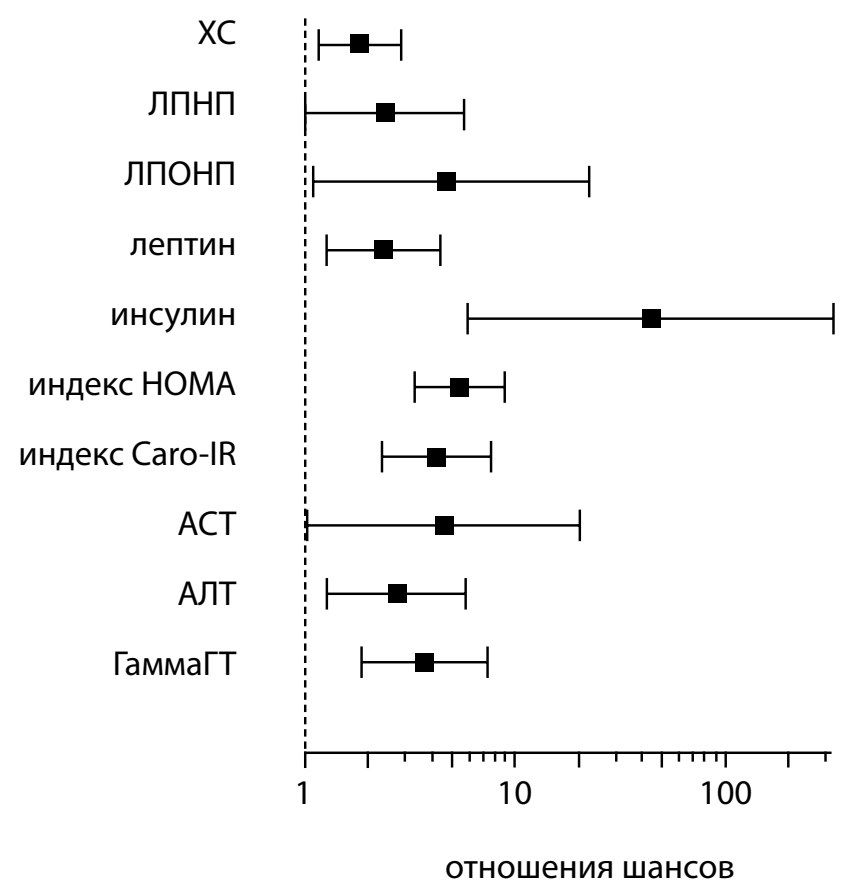

Рисунок 6. Лабораторные данные и риск метаболического нездоровья (отношение шансов) у пациентов с алиментарноконституциональным ожирением.

Далее были проанализированы основные факторы риска для диагностики метаболического нездоровья при алиментарно-конституциональном ожирении, которые представлены на рис. 4-7.

Значимым статистически достоверным фактором оказался возраст пациента старше 45 лет, так, ОШ составило 3,14 [95\% ДИ 1,877-5,254]. Также повышали вероятность МН3О мужской пол - ОШ=7,76 [95\% ДИ 1,815-33,134], отягощенная наследственность по ожирению - ОШ=1,84
OT

TГ

$\mathrm{A \Gamma}$

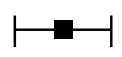

гликемия

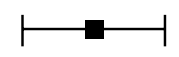

ХС лПнП
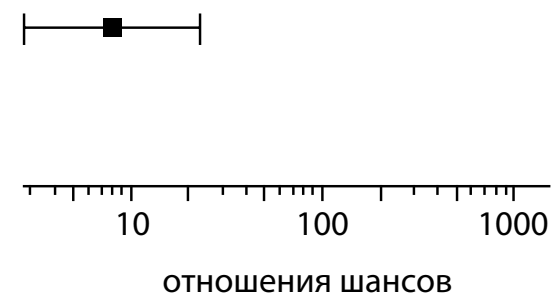

Рисунок 5. Критерии метаболического нездоровья и отношения шансов у пациентов с алиментарно-конституциональным ожирением.

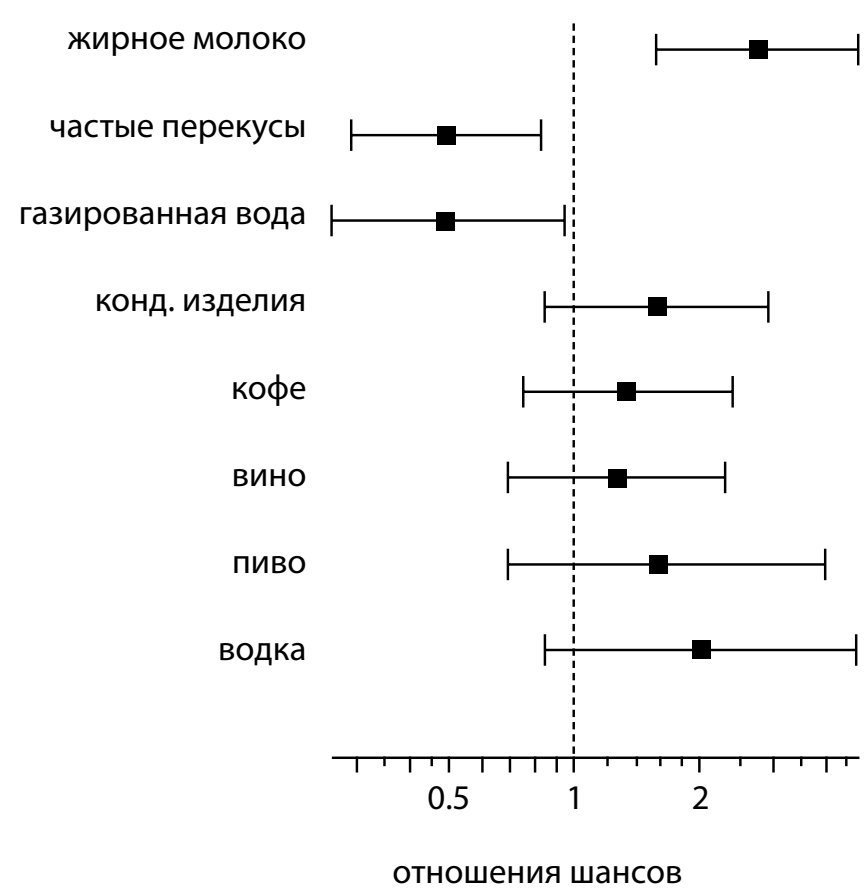

Рисунок 7. Особенности питания и риск метаболического нездоровья при алиментарно-конституциональном ожирении.

[95\% ДИ 1,079-3,133] и давность ожирения более 10 лет ОШ=3,095 [95\% ДИ 1,859-5,155]. Имели значение и ИМТ выше $35 \mathrm{kr} / \mathrm{M}^{2}$ - ОШ $=5,93$ [95\%ДИ 3,412-10,318] и ОТ/ОБОШ $=4,80$ [95\% ДИ 2,871-8,026] (см. рис. 4).

Как и следовало ожидать, наиболее высокие риски имели признаки МС, так как они легли в основу метаболического нездоровья. Однако их вклад был неодинаковым. Так, среди критериев МС самой значимой для развития метаболических нарушений была ОТ — ОШ=210,71 [95\% ДИ 
28,56-1554,09]. Также высокий риск имел уровень ТГ (ОШ=30,87 [95\% ДИ 7,40-128,70]), а меньший был связан с наличием АГ (ОШ=12,29 [95\% ДИ 6,95-21,73]), повышением уровня гликемии (ОШ=14,52 [95\% ДИ 6,14-34,31]) и низким уровнем ХС ЛПВП крови (ОШ=8,03 [95\% ДИ 2,80-23,04]) (рис. 5).

Определенные риски формирования метаболического нездоровья при ожирении были связаны с нарушением других показателей липидного профиля: повышением ХС - ОШ=1,83 [95\% ДИ 1,16-2,88], высоким уровнем ХС ЛПНП - ОШ=2,415 [95\% ДИ 1,01-5,73] и высоким уровнем ХС лПОНП - ОШ=4,67 [95\% ДИ 1,08-22,3]. Повышенный уровень лептина также увеличивал риск развития $\mathrm{MH} 3 \mathrm{O}-$ ОШ $=2,36$ [95\% ДИ 1,26-4,42]. Формирование метаболического нездоровья было связано и с наличием гиперинсулинемии и ИР. Так, при повышенном уровне инсулина крови ОШ составило 44,19 [95\% ДИ 5,98-326,65], при высоком индексе HOMA-IR - ОШ=5,44 [95\% ДИ 3,30-8,97], сниженном индексе Caro-IR - ОШ=4,20 [95\% ДИ 2,32-7,61]. Риски появления МН3О повышали и высокие уровни печеночных трансаминаз: при повышенной АСТ ОШ=4,55 [95\% ДИ 1,03-20,05], АЛТ - ОШ=2,72 [95\% дИ 1,27-5,81], Гамма-ГТ - ОШ=3,69 [95\% дИ 1,87-7,27] (рис. 6).

Проведенный анализ, в том числе корреляционный, показателей состава тела: ЖМ и БЖМ (абсолютные и относительные значения) у обследованных в возрасте старше 45 лет показал, что они не повышают риск метаболических нарушений при алиментарно-конституциональном ожирении. Однако оценка процента АКМ выявила, что 95\% пациентов с МН3О и $81 \%$ с М3О имеют этот показатель ниже $45 \%\left(X^{2}=8,86 ; p<0,005\right)$, что позволило далее его проанализировать в ОР развития метаболических нарушений при ожирении. У больных с процентом АКМ менее 45 в 1,961 раза выше ОР наличия МН3О [95\% ДИ 1,059-3,630].

Психологическое исследование не обнаружило достоверного влияния депрессивных и тревожных нарушений на риск развития МНЗО.

Также небольшое значение, по нашим данным, для формирования МН3О имели поведенческие факторы, хотя и были получены достоверные различия по многим из них (табл. 2).

Из всех изученных факторов для развития классических признаков МС имело значение только использование в пищу жирных молочных продуктов - ОШ=2,74 [95\% ДИ 1,565-4,78]. Напротив, частые перекусы (ОШ=0,49 [95\% ДИ 0,29-0,83]) и газированная вода и соки (ОШ=0,49 [95\% ДИ 0,26-0,94]) не влияли на формирование метаболических нарушений при ожирении - имеется обратная статистически значимая связь (см. рис. 4).

Достоверного риска развития МН3О не было отмечено при употреблении кондитерских изделий (ОШ=1,58 [95\% ДИ 0,85-2,92]) и кофе (ОШ=1,34 [95\% ДИ 0,75-2,40]). Потребление спиртных напитков, хотя и повышало риск МН3О, но также было не достоверно. Так, у предпочитающих вино ОШ составило 1,26 [95\% ДИ 0,69-2,29], пиво - ОШ=1,58 [95\% ДИ 0,62-3,99], при употреблении крепких напитков, в частности водки, ОШ=2,01 [95\% ДИ 0,85-4,74].
Физические нагрузки (ни частота, ни их вид) не имели достоверных рисков развития МНзо.

Семейные традиции имели риски появления метаболического нездоровья, но они были недостоверны. Так, при отсутствии режима питания в детстве ОШ составило 1,18 [95\% ДИ 0,42-3,28], при принуждении к еде ОШ=1,33 [95\% ДИ 0,43-4,07], поощрении сладостями ОШ=1,12 [95\% ДИ 0,34-3,68] (рис. 7).

\section{Нежелательные явления}

Нежелательных явлений зафиксировано не было.

\section{ОБСУЖДЕНИЕ}

Репрезентативность выборок

Выборка репрезентативна, и результаты исследования могут экстраполироваться на целевую популяцию. Никаких специфических факторов, способных повлиять на выводы работы, не было. В исследование включались пациенты, сами обратившиеся к эндокринологу на амбулаторный прием в частное лечебно-профилактическое учреждение по поводу снижения веса, а их полное обследование проводилось в современной клинике Тверского государственного медицинского университета.

\section{Резюме основного результата исследования}

Наибольший риск для появления метаболических нарушений имеют пациенты в возрасте старше 45 лет, при снижении активной клеточной массы в организме менее 45\%, давностью ожирения более 10 лет и отягощенной по данной патологии наследственностью. Мужчины почти в 8 раз чаще, чем женщины, обращаются к эндокринологу с проблемой избыточного веса, когда у них уже имеются признаки метаболического нездоровья. Среди поведенческих факторов (пищевой статус) риск метаболического нездоровья при ожирении повышают жирные молочные продукты, а частые перекусы, сладкие безалкогольные напитки, напротив, не влияют на него.

\section{Обсуждение основного результата исследования}

Ожирение как состояние избыточного накопления жировой ткани в организме ассоциировано с целым рядом серьезных патологий и повышением риска развития сердечно-сосудистых заболеваний, а также сахарного диабета 2 типа [18-22]. Однако опубликованы данные, свидетельствующие о том, что существуют формы ожирения с нормальным метаболическим статусом: без нарушений углеводного и липидного обменов, с нормальными цифрами АД [23]. Такое ожирение принято называть М3О $[24,25]$. К настоящему моменту не разработано единых дефиниций М30, а сведения о прогнозе пациентов с МЗО противоречивы [26-30]. Интересным представляется вопрос о факторах, оказывающих влияние на формирование метаболически здорового и нездорового фенотипов ожирения.

В данной работе была предпринята попытка поиска таких факторов.

Согласно нашим данным, среди признаков МС, относящихся к метаболическому нездоровью, чаще всего у пациентов с ожирением диагностируются увеличенная окружность талии (у мужчин более 102 см и у женщин 
более 88 см), повышенный уровень ТГ (более 1,7 ммоль/л) и повышение АД (более 130/85 мм рт. ст.).

Это совпадает с результатами, полученными Ротарь О.П., так, у офисных работников (у мужчин чаще, чем у женщин), имеющих ожирение и АГ, в 70\% случаев регистрируется полный МС [31].

В результате проведенного исследования было выявлено, что риск диагностики МН3О выше в возрастной группе старше 45 лет, у пациентов с отягощенным по ожирению наследственным анамнезом и при стаже ожирения более 10 лет.

Действительно, имеются данные о существенном возрастании сердечно-сосудистого риска у пациентов с длительностью М3О более 10-15 лет [32, 33]. В этих исследованиях было показано, что практически у половины пациентов изменение метаболического статуса на метаболически нездоровый было ассоциировано с увеличением показателей ИМТ, ОТ, изменением состава тела, повышением уровней инсулина, гликированного гемоглобина и липидов крови [34]. В нашей работе мы получили схожие результаты по риску МНЗО при повышении показателей антропометрии, липидов и инсулина крови, а также инсулинорезистентности. Из фракций холестерина наибольшее значение имели липопротеиды очень низкой плотности. Кроме этого, на наличие метаболического нездоровья указывали повышенные лептин крови и печеночные показатели.

Среди факторов, обладающих протективным воздействием на метаболическое здоровье, многие авторы отмечают правильное питание и высокий уровень физической активности $[35,36]$. Доказано, что образ жизни, включая пищевые привычки, играет существенную роль в сохранении здоровья [37]. Так, низкая частота аэробных нагрузок у лиц с ожирением увеличивала риск смерти в 2,5 раза [38]. Это представляется логичным в связи с тем, что физические нагрузки, особенно аэробные, приводят к уменьшению жировой массы организма. Снижение мышечной (метаболически активной ткани) и увеличение жировой массы ассоциированы со снижением основного обмена и метаболическими нарушениями. При оценке состава тела нами было выявлено, что снижение АКМ (метаболически активных тканей организма, расходующих калории) менее 45\% является фактором риска развития МH3О. В то же время в метаанализе 10 проспективных исследований были получены данные о сопоставимом риске смерти у тренированных людей как с нормальной массой тела, так и при наличии ожирения. В своем исследовании мы также не получили влияния частоты и вида физических нагрузок на метаболическое нездоровье.

Проведенные нами психологическое исследование и оценка особенностей пищевого поведения не обнаружили достоверного влияния как депрессивных и тревожных нарушений, так и особенностей пищевого поведения на риск развития МНЗО. В то же время в литературе имеются данные о коморбидности депрессии и тревоги с MC (в 62\% случаев), а также о преобладании у этих пациентов эмоционального и компульсивного типов пищевого поведения [39].

Существуют данные о роли питания в формировании МС. Исследованию в основном подвергались макронутриенты (белки, жиры и углеводы), а также отдельные типы фактического питания. Так, риск развития МС был выше в максимальном квентиле употребления углеводов $[37,40]$. Приверженность к западному типу питания (очищенные зерновые, жареные блюда, красное мясо) значимо повышает риск развития МС. В нашей работе изучение пищевых предпочтений показало, что наличие перекусов между основными приемами пищи не приводило к риску метаболических нарушений при ожирении, а вот частое употребление жирных молочных продуктов его повышало. Не оказывало влияния на появление $\mathrm{MH} 3 \mathrm{O}$ и употребление сладких безалкогольных напитков.

\section{Ограничения исследования}

В настоящее время не имеется единых общепризнанных дефиниций метаболически здорового и нездорового ожирения, в связи с чем данные о распространенности могут варьировать. Ответы на анкетирование по образу жизни носят субъективный характер.

\section{ЗАКЛЮЧЕНИЕ}

Анализ изученных рисков формирования метаболического нездоровья при алиментарно-конституциональном ожирении показал их неоднозначное влияние. Наибольшее значение для развития МН3О имеет абдоминальное ожирение (увеличение ОТ у женщин более 88 см и у мужчин более 102 см), которое тесно связано со степенью ожирения. При этом МНЗО ассоциировано не только с классическими признаками МС, но и с уровнем инсулина крови, ИР, нарушениями липидного спектра и функции печени. Наибольший риск появления метаболических нарушений имеют пациенты в возрасте старше 45 лет, при снижении АКМ в организме менее 45\%, с давностью ожирения более 10 лет и отягощенной по данной патологии наследственностью. Среди поведенческих факторов (пищевой статус) риск МН3О повышают жирные молочные продукты, а частые перекусы, сладкие безалкогольные напитки, напротив, не влияют на него.

\section{ДОПОЛНИТЕЛЬНАЯ ИНФОРМАЦИЯ}

Источники финансирования. Работа выполнена при инструментальном обеспечении клиник Тверского медицинского университета и «Восстановительной медицины».

Конфликт интересов. Авторы декларируют отсутствие явных и потенциальных конфликтов интересов, связанных с содержанием настоящей статьи.

Участие авторов. Лясникова М.Б. - получение данных, написание статьи; Белякова Н.А. - дизайн исследования, правка статьи, финальная версия статьи; Цветкова И.Г. - статистическая обработка и анализ данных, внесение правки с целью повышения её научной значимости; Родионов А.А. — концепция исследования, внесение в финальную версию статьи правки; Милая Н.О. - интерпретация результатов, подготовка материала к написанию статьи. Все авторы одобрили финальную версию статьи перед публикацией, выразили согласие нести ответственность за все аспекты работы, подразумевающую надлежащее изучение и решение вопросов, связанных с точностью или добросовестностью любой части работы.

Благодарности. Позвольте выразить благодарность руководителям и сотрудникам частной клиники «Восстановительная медицина» (главный врач - Е.Б. Максубова) г. Твери, которые явились инициаторами проведения исследования по коррекции веса и оказывали техническую и консультативную помощь при сборе материала. 


\section{СПИСОК ЛИТЕРАТУРЫ | REFERENCES}

1. Kopelman PG. Obesity as a medical problem. Nature. 2000;404(6778):635-643. doi: https://doi.org/10.1038/35007508

2. González-Muniesa P, Mártinez-González M-A, Hu FB, et al. Obesity. Nat Rev Dis Prim. 2017;3(1):17034. doi: https://doi.org/10.1038/nrdp.2017.34

3. Di Angelantonio E, Bhupathiraju SN, Wormser D, et al. Body-mass index and all-cause mortality: individualparticipant-data meta-analysis of 239 prospective studies in four continents. Lancet. 2016;388(10046):776-786. doi: https://doi.org/10.1016/S0140-6736(16)30175-1

4. Tchernof A, Després J-P. Pathophysiology of Human Visceral Obesity: An Update. Zmijewski M, ed. Physiol Rev. 2013;93(1):359-404. doi: https://doi.org/10.1152/physrev.00033.2011

5. Mayoral L-C, Andrade G, Mayoral E-C, et al. Obesity subtypes, related biomarkers \&amp; heterogeneity. Indian J Med Res. 2020;151(1):11. doi: https://doi.org/10.4103/ijmr.IJMR_1768_17

6. Goossens $\mathrm{GH}$. The role of adipose tissue dysfunction in the pathogenesis of obesity-related insulin resistance. Physiol Behav. 2008;94(2):206-218. doi: https://doi.org/10.1016/j.physbeh.2007.10.010

7. Rosen ED, Spiegelman BM. What We Talk About When We Talk About Fat. Cell. 2014;156(1-2):20-44. doi: https://doi.org/10.1016/i.cell.2013.12.012

8. Stinkens R, Goossens GH, Jocken JWE, Blaak EE. Targeting fatty acid metabolism to improve glucose metabolism. Obes Rev. 2015;16(9):715-757. doi: https://doi.org/10.1111/obr.12298

9. Wildman RP. The Obese Without Cardiometabolic Risk Factor Clustering and the Normal Weight With Cardiometabolic Risk Factor Clustering. Arch Intern Med. 2008;168(15):1617. doi: https://doi.org/10.1001/archinte.168.15.1617

10. World Health Organization. Obesity: preventing and managing the global epidemic. Geneva:WHO, 1997. Available from: http://www. who.int/nutrition/publications/obesity/WHO_TRS_894/en/

11. Expert Panel on Detection, Evaluation and T of HBC in A. Executive Summary of the Third Report of the National Cholesterol Education Program (NCEP) Expert Panel on Detection, Evaluation, and Treatment of High Blood Cholesterol in Adults (Adult Treatment Panel III). JAMA J Am Med Assoc. 2001;285(19):2486-2497. doi: https://doi.org/10.1001/jama.285.19.2486

12. Bloomgarden ZT. American Association of Clinical Endocrinologists (AACE) Consensus Conference on the Insulin Resistance Syndrome. Diabetes Care. 2003;26(3):933-939. doi: https://doi.org/10.2337/diacare.26.3.933

13. van Vliet-Ostaptchouk JV, Nuotio M-L, Slagter SN, et al. The prevalence of metabolic syndrome and metabolically healthy obesity in Europe: a collaborative analysis of ten large cohort studies. BMC Endocr Disord. 2014;14(1):9. doi: https://doi.org/10.1186/1472-6823-14-9

14. Beck AT. A systematic investigation of depression. Comprehensive Psychiatry. 1961;2(3):163-170.

15. Карелин А.А. Большая энциклопедия психологических тестов. - M.; 2007. - 416 c. [Karelin AA. Bolshaya encyklopedia psykhologicheskih testov. Moscow; 2007.416 p. (In Russ.)].

16. van Strien T, Friiters JER, Bergers GPA, Defares PB. The Dutch Eating Behavior Questionnaire (DEBQ) for assessment of restrained, emotional, and external eating behavior. Int J Eat Disord. 1986;5(2):295-315. doi: https://doi.org/10.1002/1098108X(198602)5:2<295::AID-EAT2260050209>3.0.CO;2-T

17. Белякова Н.А., Милая Н.О., Лясникова М.Б. Анкетирование по питанию и физическим нагрузкам. Свидетельство на НОУ-ХАУ, регистрационный номер 01-126. от 28.06.2019. [Belyakova NA, Milaya NO, Lyasnikova MB. Anketirovanie po pitaniyu i fizicheskim nagruzkam. Registratsionnyi nomer 01-126, data registratsii 28.06.2019. (In Russ.)].

18. Морбидное ожирение / Под ред. Дедова И.И. - М.: Издательство «Медицинское информационное агентство»; 2014. - 605 с [Morbidnoe ozhirenie. Ed. by Dedov II. Moscow: Izdatel'stvo «Meditsinskoe informatsionnoe agentstvo»; 2014.605 p. (In Russ.)].

19. Piché $M-E$, Tchernof $A$, Després J-P. Obesity Phenotypes, Diabetes, and Cardiovascular Diseases. Circ Res. 2020;126(11):1477-1500. doi: https://doi.org/10.1161/CIRCRESAHA.120.316101

20. Seravalle G, Grassi G. Obesity and hypertension. Pharmacol Res. 2017;122:1-7. doi: https://doi.org/10.1016/j.phrs.2017.05.013
21. Vecchié A, Dallegri F, Carbone F, et al. Obesity phenotypes and their paradoxical association with cardiovascular diseases. Eur Intern Med. 2018;48:6-17. doi: https://doi.org/10.1016/j.ejim.2017.10.020

22. Pischon T, Nimptsch K. Obesity and Risk of Cancer: An Introductory Overview. In: Recent Results in Cancer Research. 2016;208:1-15. doi: https://doi.org/10.1007/978-3-319-42542-9_1

23. Jung C.H. Metabolically healthy obesity: a friend or foe? Korean J Intern Med. 2017;32(4):611-621. doi: https://doi.org/10.3904/kjim.2016.259

24. Романцова Т.И., Островская Е.В. Метаболически здоровое ожирение: дефиниции, протективные факторы, клиническое значение // Альманах клин мед. - 2015. - Т. 61. - С. 75-86. [Romantzova T.I. Ostrovskaya E.V. Metabolicheski zdorovoye ozhirenie: definitzii, protectivniye faktory, klinicheskoye znachenije. Almanah klin med. 2015;51:75-86 (In Russ.)].

25. Островская Е.В., Романцова Т.И., Герасимов А.Н., Новоселова Т.Е. Распространенность метаболически здорового ожирения по данным выборки Московского региона // Ожирение и метаболизм. - 2017. - Т. 14. №4. - C. 51-56. [Ostrovskaja EV, Romancova TI, Gerasimov AN, Novoselova TE. Rasprostranennost' metabolicheski zdorovogo ozhirenija po dannym vyborki Moskovskogo regiona. Ozhirenie i metabolizm. 2017;14(4):51-56. (In Russ.)]. doi: https://doi.org/10.14341/omet2017451-56

26. Al Suwaidi J. Is there an increased cardiovascular risk in metabolically healthy obese individuals? Lessons from the HUNT (NordTrøndelag Health) study. Glob Cardiol Sci Pract. 2014;2014(2):24. doi: https://doi.org/10.5339/gcsp.2014.24

27. Meigs JB, Wilson PWF, Fox CS, et al. Body Mass Index, Metabolic Syndrome, and Risk of Type 2 Diabetes or Cardiovascular Disease. J Clin Endocrinol Metab. 2006;91(8):2906-2912. doi: https://doi.org/10.1210/jc.2006-0594

28. Luo D, Liu F, Li X, et al. Comparison of the effect of 'metabolically healthy but obese' and 'metabolically abnormal but not obese' phenotypes on development of diabetes and cardiovascular disease in Chinese. Endocrine. 2015;49(1):130-138 doi: https://doi.org/10.1007/s12020-014-0444-2

29. Appleton SL, Seaborn CJ, Visvanathan R, et al. Diabetes and Cardiovascular Disease Outcomes in the Metabolically Healthy Obese Phenotype. Diabetes Care. 2013;36(8):2388-2394. doi: https://doi.org/10.2337/dc12-1971

30. Soriguer F, Gutiérrez-Repiso C, Rubio-Martín E, et al. Metabolically Healthy but Obese, a Matter of Time? Findings From the Prospective Pizarra Study. J Clin Endocrinol Metab. 2013;98(6):2318-2325. doi: https://doi.org/10.1210/jc.2012-4253

31. Ротарь О.П. Распространенность кардиометаболических нарушений, поражения органов мишеней и механизм их прогрессирования у офисных работников. Дис. ... д-ра мед. наук. - СПб.; 2016. 221 с. [Rotarh OP. Rasprostranyonnost kardiometabolitcheskih narushenij, porazheniya organov misheney i mekhanizm ih progressirovaniya u ofisnykh rabotnokov. [dissertation]. Saint-Petersburg; 2016. 221 p. (In Russ.)].

32. Kramer CK, Zinman B, Retnakaran R. Are Metabolically Healthy Overweight and Obesity Benign Conditions? Ann Intern Med. 2013;159(11):758-769. doi: https://doi.org/10.7326/0003-4819-159-11-201312030-00008

33. Fan J, Song Y, Chen Y, et al. Combined effect of obesity and cardio-metabolic abnormality on the risk of cardiovascular disease: A meta-analysis of prospective cohort studies. Int J Cardiol. 2013;168(5):4761-4768. doi: https://doi.org/10.1016/j.ijcard.2013.07.230

34. Hamer M, Bell JA, Sabia S, et al. Stability of metabolically healthy obesity over 8 years: the English Longitudinal Study of Ageing. Eur J Endocrinol. 2015;173(5):703-708. doi: https://doi.org/10.1530/EJE-15-0449

35. Eshtiaghi R, Keihani S, Hosseinpanah F, et al. Natural course of metabolically healthy abdominal obese adults after 10 years of follow-up: the Tehran Lipid and Glucose Study. Int J Obes. 2015;39(3):514-519. doi: https://doi.org/10.1038/ijo.2014.176

36. Hwang Y-C, Hayashi T, Fujimoto WY, et al. Visceral abdominal fat accumulation predicts the conversion of metabolically healthy obese subjects to an unhealthy phenotype. Int $J$ Obes. 2015;39(9):1365-1370. doi: https://doi.org/10.1038/ijo.2015.75 
37. Heidemann C, Scheidt-Nave C, Richter A, Mensink GBM. Dietary patterns are associated with cardiometabolic risk factors in a representative study population of German adults. Br J Nutr. 2011;106(8):1253-1262. doi: https://doi.org/10.1017/S0007114511001504

38. Barry VW, Baruth M, Beets MW, et al. Fitness vs. Fatness on All-Cause Mortality: A Meta-Analysis. Prog Cardiovasc Dis. 2014;56(4):382-390. doi: https://doi.org/10.1016/j.pcad.2013.09.002
39. Успенский Ю.П., Петренко Ю.В., Гулунов 3.Х., и др. Метаболический синдром. Учебное пособие. — СПб; 2017. — 60 c. [Uspenskij YP, Petrenko YV, Gulunov ZH, et al. Metabolitchesky syndrom. Uchebnoye posobiye. $\mathrm{SPb}$.; 2017.60 p. (in Russ.)].

40. Lutsey PL, Steffen LM, Stevens J. Dietary Intake and the Development of the Metabolic Syndrome. Circulation. 2008;117(6):754-761. doi: https://doi.org/10.1161/CIRCULATIONAHA.107.716159

\section{ИНФОРМАЦИЯ ОБ АВТОРАХ [AUTHORS INFO]:}

*Белякова Наталья Александровна, д.м.н., професcop [Nataliya A. Belyakova, MD, PhD, Professor]; адрес: Россия, Тверь, 170100, ул. Советская, д. 4 [address: 4 Sovetskay street, 170100 Tver, Russia]; ORCID: https://orcid.org/0000-0003-4465-2505; eLibrary SPIN: 4357-5266; e-mail: tverendo@mail.ru

Лясникова Мария Борисовна, к.М.н., доцент [Mariya B. Lyasnikova, MD, PhD]; ORCID: https://orcid.org/0000-0003-4784-4107; eLibrary SPIN: 2794-6812; e-mail: mashulyasnik@mail.ru

Цветкова Инна Геннадьевна, к.м.н., доцент [Inna G. Tsvetkova, MD, PhD]; ORCID: https://orcid.org/0000-0002-0306-0848; eLibrary SPIN: 1152-8179; E-mail: inna-zvetkova@mail.ru

Родионов Андрей Александрович, к.м.н. [Andrey A. Rodionov, MD, PhD]; ORCID: 0000-0002-7226-772X; eLibrary SPIN: 8175-4965; e-mail: andrew.rodionov@tvergma.ru

Милая Наталия Олеговна, К.M.н. [Nataliya O. Milaya, PhD]; ORCID: https://orcid.org/0000-0001-8169-4187;

eLibrary SPIN: 5120-1188; E-mail: nataliya.suslikova.88@mail.ru

*Автор, ответственный за переписку / Corresponding author.

\section{ЦИТИРОВАТЬ:}

Лясникова М.Б., Белякова Н.А., Цветкова И.Г., Родионов А.А., Милая Н.О. Риски развития метаболического нездоровья при алиментарно-конституциональном ожирении // Ожирение и метаболизм. — 2021. — Т. 18. — №4. — С. $406-416$. doi: https://doi.org/10.14341/omet12705

\section{TO CITE THIS ARTICLE:}

Lyasnikova MB, Belyakova NA, Tsvetkova IG, Rodionov AA, Milaya NO. Risks for development of metabolic disorders in alimentary constitutional obesity. Obesity and metabolism. 2021;18(4):406-416. doi: https://doi.org/10.14341/omet12705 